\title{
Toxicity and quality of life report of a phase II study of stereotactic body radiotherapy (SBRT) for low and intermediate risk prostate cancer
}

Matthew J. Boyer ${ }^{1 *}$, Michael A. Papagikos ${ }^{2}$, Rex Kiteley ${ }^{3}$, Zeljko Vujaskovic ${ }^{4}$, Jackie Wu ${ }^{1}$ and W. Robert Lee ${ }^{1}$

\begin{abstract}
Background: Clinical data indicates that delivery of larger daily doses of radiation may improve the therapeutic ratio for prostate cancer compared to conventional fractionation. A phase II study of stereotactic body radiotherapy with real-time motion management and daily plan re-optimization for low to intermediate risk prostate cancer was undertaken to evaluate this hypothesis. This report details the toxicity and quality of life following treatment.

Methods: From 2009 to 2013, 60 patients with T1-T2C prostate cancer with a Gleason score of 6 and PSA $\leq 15$ or Gleason score of 7 and PSA $\leq 10$ were enrolled. Patients with nodal metastases, an American Urological Association symptom score $>18$, or gland size $>100 \mathrm{~g}$ were not eligible. Patients were treated to $37 \mathrm{~Gy}$ in 5 fractions. Early and late genitourinary and gastrointestinal toxicity were graded based on NCI CTCAE v4.0 and quality of life was assessed by the American Urological Association symptom score, International Index of Erectile Function, and Expanded Prostate cancer Index Composite Short Form up to 36 months after treatment.

Results: After a median follow-up of 27.6 months, no grade 3 or greater genitourinary toxicity was observed. Four patients (6.7\%) reported a late grade 2 genitourinary toxicity. One patient (1.7\%) reported a late grade 3 gastrointestinal toxicity. Five patients (8.3\%) developed a late grade 2 gastrointestinal toxicity. The median American Urological Association symptom score increased from 4.5 prior to treatment to 11 while on treatment $(p<0.01)$, but was 5 at 36 months post-treatment $(p=0.65)$. Median International Index of Erectile Function scores decreased from 19 to 17 over the course of follow-up $(p<0.01)$. Only median scores within the Expanded Prostate Cancer Index Composite Short Form sexual domain were significantly decreased at 36 months post-treatment (67.9 vs $45.2, p=0.02$ ). There was no significant difference in median score within the urinary, bowel, or hormonal domains at 36 months of follow-up.
\end{abstract}

Conclusions: Stereotactic body radiotherapy for low to intermediate risk prostate cancer is well tolerated with limited toxicity or decrease in quality of life. Longer follow-up is necessary to assess the efficacy of treatment.

Trial registration: Clinicaltrials.gov NCT00941915 Registered 17 June 2009.

Keywords: Prostate cancer, Stereotactic body radiation therapy, Toxicity, Quality of life

\footnotetext{
*Correspondence: matthew.boyer@dm.duke.edu

'Department of Radiation Oncology, Duke University Medical Center, DUMC

Box 3085, Durham, NC 27710, USA

Full list of author information is available at the end of the article
} 


\section{Background}

Conventional treatment of localized prostate cancer with radiation alone involves doses to 74 Gy or greater given over 8 to 9 weeks. This regimen is based on four randomized trials showing improved progression free survival compared to lower cumulative doses, however at the cost of increased toxicity [1-4]. Given this concomitant increase in toxicity with dose, as well as the expense and inconvenience of protracted courses, alternative treatment schemes have been investigated.

The relationship of cellular death to radiation dose for rapidly dividing cells is dominated by a linear component, represented by a large $\alpha / B$ ratio, such that changes in fraction size have a small impact on efficacy. More slowly dividing cells, with a relatively small $\alpha / \beta$ are more sensitive to changes in fraction size when delivered to equivalent total doses. There is clinical data $[5,6]$ that suggests that prostate cancer has a low $\alpha / \beta$ compared to surrounding normal tissues and therefore increasing daily radiation fraction size will have a greater effect on the tumor and increase the therapeutic ratio. Hypofractionation, or the delivery of fewer, larger fractions to a lower total dose, may allow for increased tumor control and limit the toxicity and practical disadvantages of dose escalation.

Stereotactic body radiation therapy (SBRT) allows for the delivery of large radiation doses through incorporation of coordinate systems and accounting for organ motion via daily imaging. Previous reports have demonstrated the feasibility of this technique and high rates of intermediate term biochemical control for patients with low and intermediate risk prostate cancer [7-12]. Here, we report the acute and long-term toxicity of a phase II, multi-institutional study of SBRT for low to intermediate risk prostate cancer.

\section{Methods}

\section{Patients and eligibility criteria}

Men over the age 40 with prostate adenocarcinoma with a Gleason score $\leq 7$, clinical stage $\mathrm{T} 1-\mathrm{T} 2 \mathrm{c}$, and $\mathrm{PSA} \leq$ $15 \mathrm{ng} / \mathrm{ml}$ if their Gleason score was $\leq 6$ or PSA $\leq 10 \mathrm{ng} / \mathrm{ml}$ if their Gleason score was seven were eligible for this study. Other eligibility criteria included a pathological diagnosis within 365 days of enrollment, history and physical exam including digital rectal exam within 8 weeks of enrollment, and a Zubrod performance status of 0-1. Exclusion criteria included node positive or metastatic disease, AUA score $>18$, prostate size $>100 \mathrm{~g}$, previous surgery for prostate cancer, prior pelvic irradiation, or previous or concurrent androgen deprivation therapy.

Patients were recruited at three centers: Duke University Medical Center in Durham, NC, Coastal Carolina Radiation Oncology in Wilmington, NC, and Walter Reed National Military Medical Center in Bethesda, MD. Institutional review board approval was obtained at each center and all participants provided written informed consent before registration.

\section{Treatment planning}

The clinical target volume (CTV) was the prostate as determined by planning CT scan with 1 to $1.25 \mathrm{~mm}$ slice thickness without contrast. All patients completed a bladder and rectal preparation protocol including 1 tablespoon of milk of magnesia the evenings prior to simulation and treatment and a Fleet's enema with $16-24$ oz of water $2-3$ $\mathrm{h}$ before simulation and treatments. This was shown to minimize intra-fraction motion based on analysis of the first 15 treated patients. Based on this minimal motion, planning based on fused CT/MRI image sets was allowed after this time. The seminal vesicles were not included in the CTV as the risk of this in patients with a combined Gleason score of seven but primary Gleason score of three has been shown to be $4 \%$ [13]. The planning target volume (PTV) was created by expanding the CTV by $5 \mathrm{~mm}$ in all directions except by $3 \mathrm{~mm}$ posteriorly [9]. The total dose was prescribed to cover $\geq 95 \%$ of the PTV via intensity modulated radiotherapy (IMRT) with a minimum of seven non-opposed fields or two arc rotational fields. For IMRT, fluence mapping of each field was optimized via minimizing a cost function combining PTV coverage and OAR sparing followed by a leaf-motion optimization that converts the fluence map in to a sliding window delivery. And for rotational fields, a similar cost function was used for the optimization, where gantry positions, MLC leaf positions and dose at each gantry position were optimized.

The maximum doses to the rectum and bladder were 105 and $110 \%$ of the prescription dose, respectively. Fifty percent of the rectum was limited to $\leq 24 \mathrm{~Gy}$ and up to $40 \mathrm{cc}$ of bladder could receive $>24 \mathrm{~Gy}$. The maximum doses to the penile bulb and femoral heads were 100 and $81 \%$, respectively. Isodose curves at mid-prostate for a representative patient are shown in Fig. 1. All plans underwent QA check by principal investigator (WRL) and lead physicist (JW).

\section{Radiation delivery}

Patients were treated SBRT with 6 to $15 \mathrm{MV}$ beams to a total dose of 37 Gy to cover at least $95 \%$ of the PTV volume in 5 fractions. Although not based on an external coordinate system, the term SBRT was used in order to be consistent with CPT coding. Treatment machine QA was performed daily and monthly and patient specific QA performed prior to treatment. The dose was selected as an intermediate between among fractionation schedules published at the time of the study design [9-12]. Stereotactic treatment was accomplished with either implanted transponders (Calypso) or ExacTrac system and/or cone beam CT with fiducial markers. $68 \%$ of patients were treated using Calypso transponders. Each of the three institutions were required to verify that the margins were sufficient 

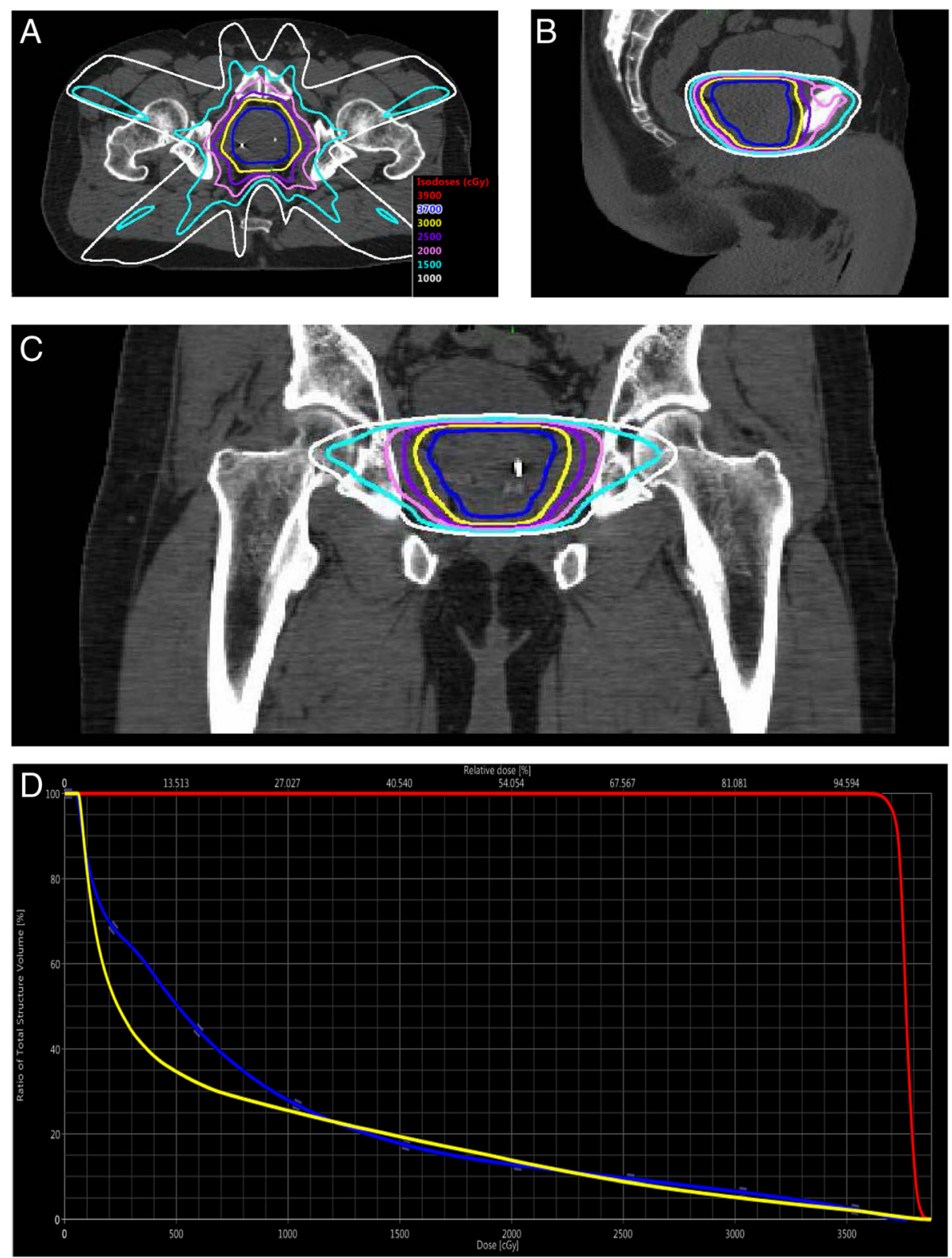

Fig. 1 Representative treatment plan. Isodose curves for a representative 7 field treatment plan in the axial (a), sagittal (b), and coronal (c) planes. d Dose volume histogram of this plan with PTV in red, rectum in blue, and bladder in yellow

using the technologies in place at each center be it Calypso, ExacTrac or CBCT. Treatment was delivered every other day with a minimum of $36 \mathrm{~h}$ to a maximum of $96 \mathrm{~h}$ between consecutive treatments. The total duration of treatment was between 10 and 18 days.

\section{Toxicity and quality of life assessment}

Toxicity, as defined by NCI CTCAE v4.0, was assessed at follow-up visits $1,3,6,12,18,24$, and 36 months following the end of treatment. Acute toxicity was defined as occurring within 90 days of completing treatment. American Urological Association Symptom Scores
(AUASS) [14] and International Index of Erectile Function (IIEF) [15] scores were collected once prior to treatment and then following treatment at the above time points via questionnaires. Expanded Prostate Cancer Index Composite Short Form (EPIC-26) bowel, urinary, hormonal, and sexual quality of life (QOL) scores [16] were obtained by questionnaire once prior to treatment and at 3, 12, 24, and 36 month follow-up appointments.

\section{Statistical methods}

The primary endpoint of this phase II study was the incidence of acute and late genitourinary (GU) and 
gastrointestinal (GI) toxicity with the null hypothesis that SBRT is not tolerable. Secondary endpoints included disease-free survival and patient QOL. Estimated grade 3 rectal toxicity for this regimen was $1.5 \%$ based on a $\mathrm{BED}_{3 \mathrm{~Gy}}$ of $78 \mathrm{~Gy}$ in 39 fractions. With 60 patients there would be $77 \%$ power to rule out a $>7.1 \%$ rate of late Grade 3 toxicity. A rate of $\geq 20 \%$ of grade 3 or greater GU or GI toxicity was considered unacceptable and $5 \%$ acceptable. Interim analyses of the rates of toxicity were planned after 15 and 30 analyzable patients were recruited.

Frequency distributions of patient demographics and grade 0 to $5 \mathrm{GU}$ and GI toxicity were compared using $\mathrm{X}^{2}$ tests. Actuarial toxicity rates were calculated using the Kaplan-Meier method. QOL scores were normalized to baseline value prior to treatment. The Wilcoxon ranked sign test was used to analyze the change in QOL scores with time. Median follow-up time was computed from the end of treatment to the last follow-up date.

\section{Results}

\section{Patient demographics}

From November 2009 through December of 2013, 60 patients were enrolled. The median age was 66. Median pre-treatment PSA was 5.8. $11(18 \%)$ patients had T2a disease, two (3\%) had T2b disease, and the remainder had T1c disease. Twenty- four (40\%) patients had a Gleason score of 6 and 36 (60\%) had a Gleason score of 7. Twenty (33\%) had low risk disease and 40 (67\%) had intermediate risk disease. The median follow-up time was 27.6 months (Interquartile range 25-37.0 months). Patient demographics are summarized in Table 1.

\section{GU toxicity}

No grade 3 or greater acute or late GU toxicity was observed (Table 2). Fifteen (25\%) patients developed at least one acute grade $2 \mathrm{GU}$ toxicity with 11 of these patients having urinary frequency. Five patients reported grade 2 urinary urgency, three reported grade 2 urinary retention, and two reported grade 2 urinary tract pain. Four (6.7\%) patients reported one grade 2 late GU toxicity (hematuria, urinary incontinence, retention, or urgency). The actuarial rate of late grade 2 or greater toxicity was $5.0 \%(95 \% \mathrm{CI} 0-10.5 \%)$ at 2 years (Fig. 2a).

\section{Gl toxicity}

There was one grade 3 late GI toxicity (fecal urgency), otherwise there was no early or late grade 3 or greater GI toxicity (Table 2). Three (5\%) patients developed acute grade 2 GI toxicity and $5(8.3 \%)$ patients developed late grade 2 GI toxicity. The most common GI toxicity was rectal hemorrhage. The actuarial rate of late grade 2 or greater toxicity was $10 \%$ (95\% CI $2.4-17.5 \%)$ at 2 years (Fig. 2b).
Table 1 Baseline characteristics of patients

\begin{tabular}{ll}
\hline Characteristic & \\
\hline Age, Median (Range) & $66(49-86)$ \\
Race, Number (Percentage) & $41(68.3)$ \\
White & $17(28.3)$ \\
Black & $1(1.7)$ \\
Asian & $1(1.7)$ \\
Native American & $5.83(0.5-13.53)$ \\
PSA, Median (Range) & \\
Stage, Number (Percentage) & $47(78.3)$ \\
T1c & $11(18.3)$ \\
T2a & $2(3.3)$ \\
T2b & $12(2-26)$ \\
Number of Biopsy Cores, Median (Range) & $3(1-10)$ \\
Number of Positive Cores, Median (Range) & \\
Gleason Score, Number (Percentage) & $24(40)$ \\
6 & $36(60)$ \\
7 & 27.6 \\
Median Follow-Up, Months &
\end{tabular}

\section{Quality of life}

Quality of life up to 36 months from the completion of treatment was assessed by the AUASS, IIEF, and EPIC26 scores. The median AUASS more than doubled from 4.5 at baseline to $11(p<0.01)$ during treatment, however the median AUASS of 5 at 36 months posttreatment approximated the pre-treatment baseline ( $p=$ 0.65 , Fig. 3a). IIEF scores were significantly worse at 3 (median IIEF score 18, $p=0.03$ ) and 36 months (median IIEF score $17, p=0.01$ ) post-treatment compared to the pre-treatment baseline however the median score only decreased by 2 from 19 to 17 over this time (Fig. 3b). Consistent with the results of the AUASS, EPIC-26 scores in the urinary domain were significantly decreased from a median baseline of 94.4 pre-treatment to 91.7 at 3 months $(p<0.01)$ and 88.9 at 12 months $(p<$ 0.04) following treatment but returned to baseline at later timepoints (median score of 96.3 at 36 months, $p=$ 0.65, Fig. 4a). Median EPIC-26 scores in the sexual domain were significantly decreased at $24(57.1, p<0.01)$ and $36(54.2, p=0.02)$ months post-treatment compared to a pre-treatment median of 67.8 although the number of responses returned at these timepoints were low (Fig. 4b). There was no significant decrease in the 36 month follow-up median EPIC-26 scores in either the bowel $(p=0.22)$ or hormonal $(p=0.53)$ domains. (Fig. 4c-d).

\section{Discussion}

In this report of a phase II multi-institutional study of SBRT for low to intermediate risk prostate cancer low 
Table 2 Crude toxicity rates

\begin{tabular}{|c|c|c|c|c|c|c|c|}
\hline \multicolumn{4}{|c|}{ Genitourinary Toxicity } & \multicolumn{4}{|c|}{ Gastrointestinal Toxicity } \\
\hline \multicolumn{2}{|l|}{ Acute } & \multicolumn{2}{|l|}{ Late } & \multicolumn{2}{|l|}{ Acute } & \multicolumn{2}{|l|}{ Late } \\
\hline Grade & Number (\%) & Grade & Number (\%) & Grade & Number (\%) & Grade & Number (\%, \\
\hline 1 & $44(73.3)$ & 1 & $22(36.7)$ & 1 & $19(31.7)$ & 1 & $15(25.0)$ \\
\hline 2 & $15(25.0)$ & 2 & $4(6.7)$ & 2 & $3(5.0)$ & 2 & $5(8.3)$ \\
\hline 3 & $0(0)$ & 3 & $0(0)$ & 3 & $0(0)$ & 3 & $1(1.7)$ \\
\hline 4 & $0(0)$ & 4 & $0(0)$ & 4 & $0(0)$ & 4 & $0(0)$ \\
\hline 5 & $0(0)$ & 5 & $0(0)$ & 5 & $0(0)$ & 5 & $0(0)$ \\
\hline
\end{tabular}

Rates of early, occurring $<90$ days from the end of treatment, and late, occurring $>90$ days from the end of treatment, toxicity

rates of genitourinary and gastrointestinal toxicity were observed with little change in QOL by AUASS as well as IIEF and EPIC-26 scores.

Clinical data $[5,6]$ suggest that hypofractionated radiation for prostate cancer may improve the therapeutic ratio for this disease. This data has prompted three non-inferiority studies in the last decade investigating treatment in 19 to 28 fractions compared to 39 or more [17-19]. Two of these studies have recently demonstrated hypofractionated treatment to be non-inferior to conventional fractionation with
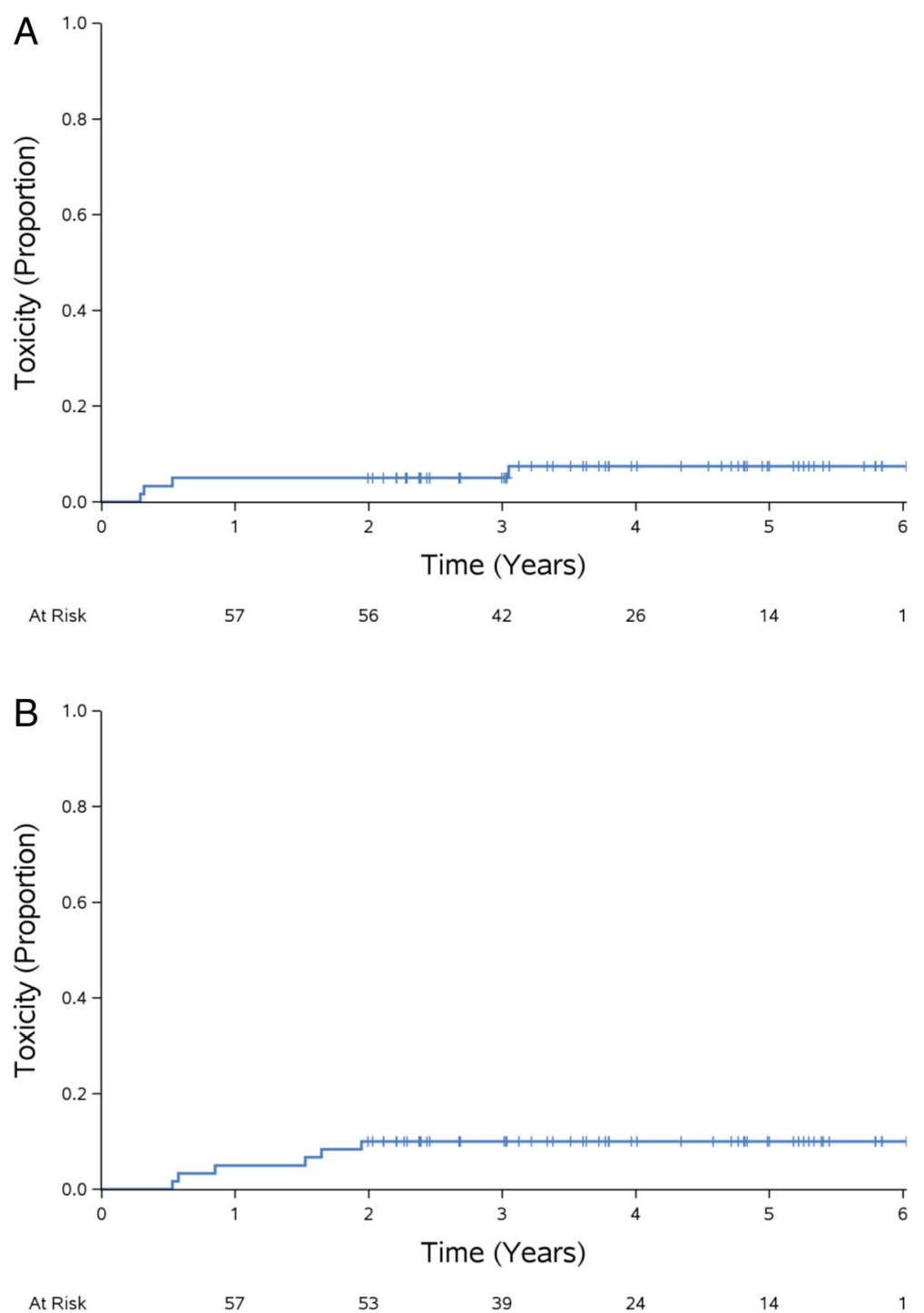

Fig. 2 Actuarial rates of toxicity. Actuarial rates of grade 2 or greater late genitourinary (a) and gastrointestinal (b) toxicity 


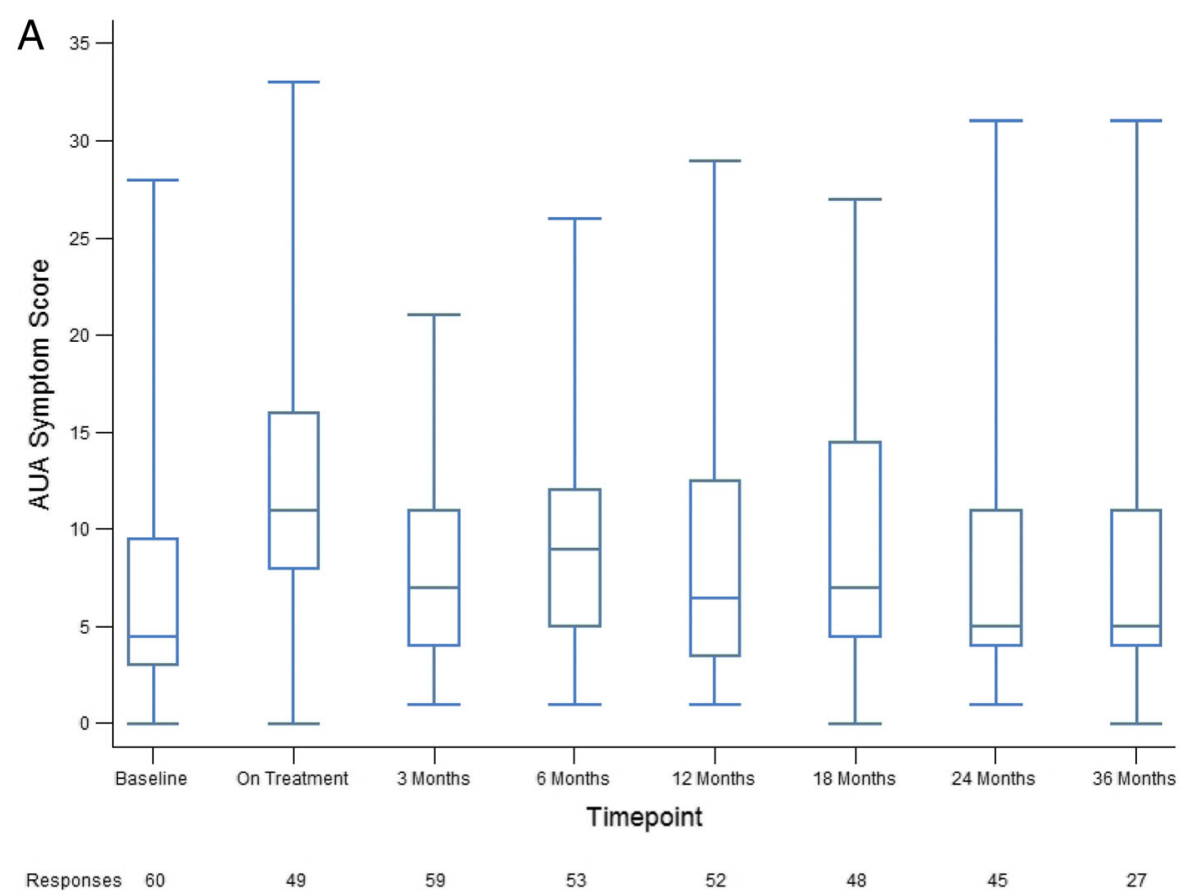

B

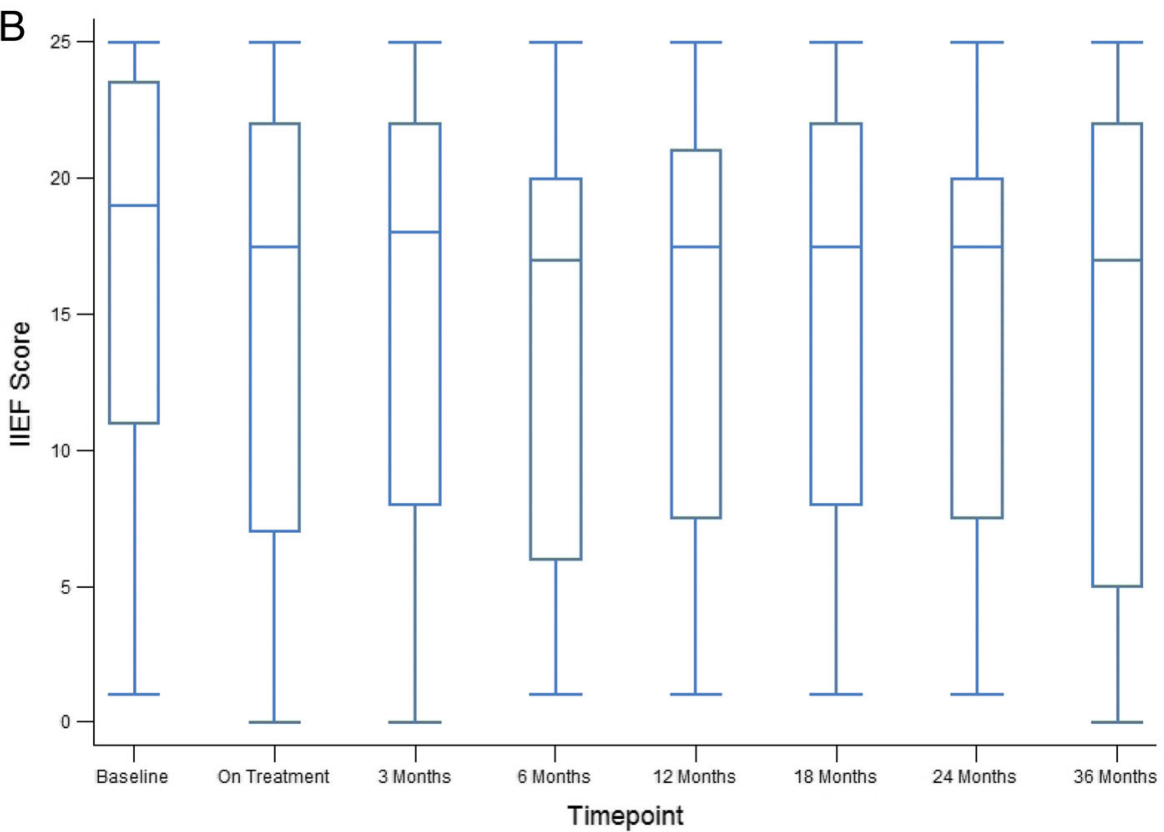

Responses 60

50

59

53

52

48

44

26

Fig. 3 Patient reported QOL scores following treatment. AUASS (a) and IIEF (b) scores at baseline, on treatment, and 3, 6, 12, 18, 24, and 36 months post-treatment. The number of responses obtained at each time point is indicated

the best estimate hazard ratios for disease free survival favoring hypofractionation $[18,19]$.

At the same time encouraging results with SBRT for sites outside of the prostate have generated enthusiasm in extending this technique to prostate cancer patients.
To this end a number of other phase I and/or II trials have been initiated to demonstrate the feasibility and tolerability of SBRT for prostate cancer.

The observed toxicity of these trials is summarized in Table 3. Consistent with these prior findings, of the 60 


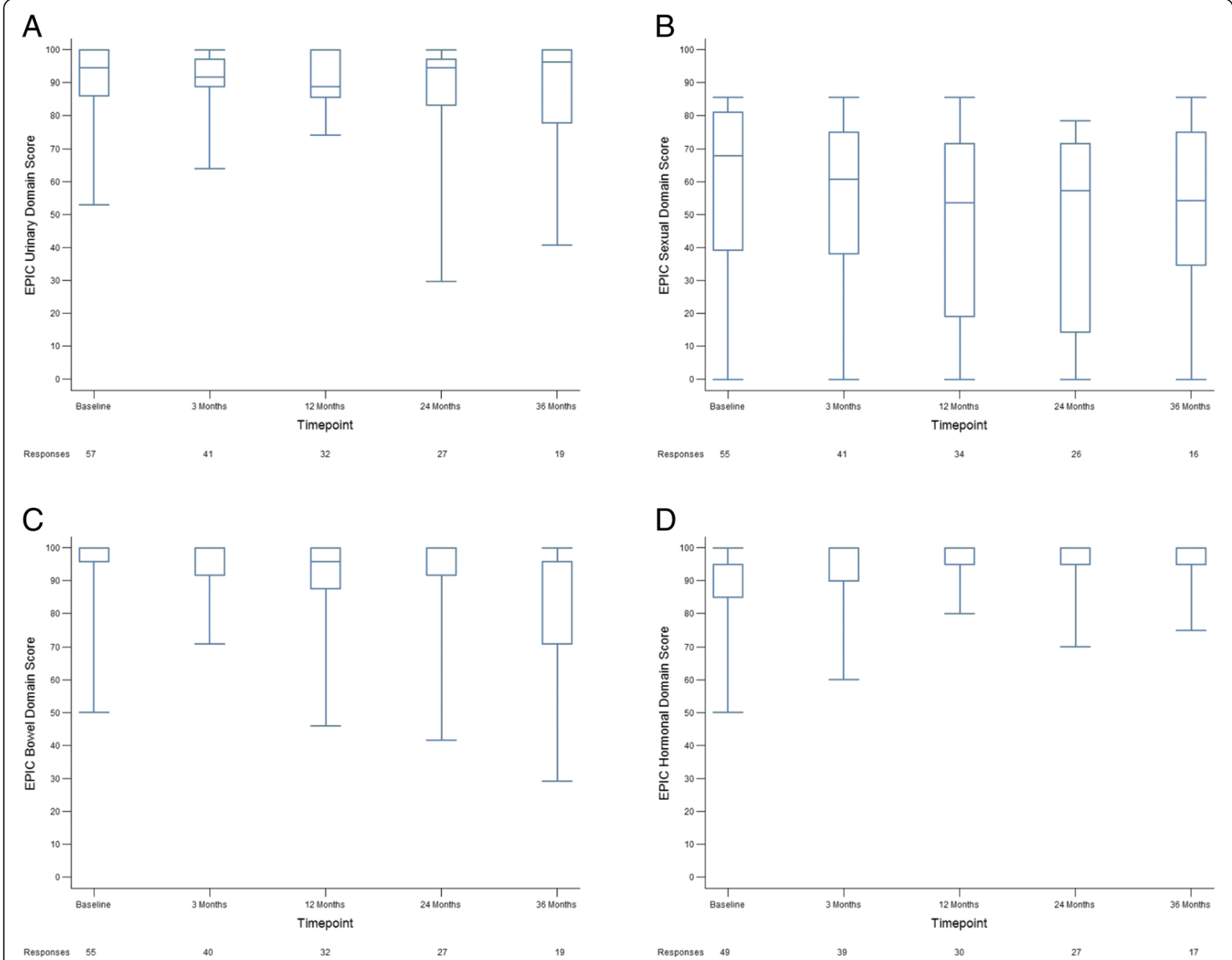

Fig. 4 Prostate cancer treatment specific QOL scores following treatment. EPIC-26 scores in the urinary (a), sexual (b), bowel (c), and hormonal (d) domains at baseline and 3, 12, 24, and 36 months post-treatment. The number of responses obtained at each time point is indicated

Table 3 Previously published rates of toxicity following prostate SBRT

\begin{tabular}{|c|c|c|c|c|c|c|c|}
\hline \multirow[t]{2}{*}{ Study } & \multirow[t]{2}{*}{$\mathrm{n}$} & \multirow{2}{*}{$\begin{array}{l}\text { Dose/ } \\
\text { Fractions }\end{array}$} & \multirow[t]{2}{*}{ Timepoint } & \multicolumn{2}{|c|}{ Genitourinary Toxicity } & \multicolumn{2}{|c|}{ Gastrointestinal Toxicity } \\
\hline & & & & Grade 2 & $\geq$ Grade 3 & Grade 2 & $\geq$ Grade 3 \\
\hline \multirow[t]{2}{*}{ Madsen [10] } & 40 & $33.5 / 5$ & Within or after 1 month & Acute $20.5 \%$ & Acute $2.5 \%$ & Acute $13 \%$ & Acute $0 \%$ \\
\hline & & & & Late $20 \%$ & Late $0 \%$ & Late $7.5 \%$ & Late $\%$ \\
\hline Tang [12] & 30 & $35 / 5$ & $\leq 6$ months & $13 \%$ & $0 \%$ & $7 \%$ & $0 \%$ \\
\hline King [9] & 41 & $36.25 / 5$ & $\geq 6$ months & $24 \%$ & $5 \%$ & $15 \%$ & $0 \%$ \\
\hline \multirow[t]{2}{*}{ McBride [11] } & 34 & $37.5-36.25 / 5$ & Within or after 3 months & Acute 19\% & Acute $0 \%$ & Acute $7 \%$ & Acute 0\% \\
\hline & & & & Late $17 \%$ & Late $2 \%$ & Late $7 \%$ & Late $5 \%$ \\
\hline \multirow[t]{2}{*}{ Hannan [8] } & 91 & $45-50 / 5$ & Within or after 9 months & Acute $22 \%$ & Acute $0 \%$ & Acute $20.9 \%$ & Acute $2.2 \%$ \\
\hline & & & & Late $20.9 \%$ & Late $5.5 \%$ & Late $13.2 \%$ & Late $6.6 \%$ \\
\hline \multirow[t]{2}{*}{ Alonghi [7] } & 40 & $35 / 5$ & Within or after 6 months & Acute $40 \%$ & Acute $0 \%$ & Acute $10 \%$ & Acute $0 \%$ \\
\hline & & & & Late $2.5 \%$ & Late $0 \%$ & Late $0 \%$ & Late $0 \%$ \\
\hline
\end{tabular}

Reported toxicity of previously published phase I and/or II trials of SBRT for prostate cancer; $n=$ number of patients on trial, dose is reported in Gy 
patients enrolled on this study only one case of grade 3 gastrointestinal toxicity was observed and no grade 3 or greater genitourinary toxicity was reported. It is possible however that late GU side effects may be underestimated due to the lack of identification of the intraprostatic urethra by imaging or introduction fo a Foley catheter at the time of simulation. There were very similar rates of acute grade $2 \mathrm{GU}$ and GI toxicity ( 25 and $5 \%$, respectively) in this study as seen in RTOG 04-15 with either hypofractionated (24.7 and 9.7\%, respectively) or conventionally fractionated (23.7 and $9.9 \%$, respectively) treatment [18]. Late grade 3 or greater toxicity was rare in this study (1 case of grade 3 GI toxicity) as well as other trials of SBRT (Table 3) or RTOG 04-15 where all rates were $6.6 \%$ or less $[7-12,18]$. A preliminary report of a randomized phase II trial, RTOG 0938, comparing SBRT in 36.25 Gy in 5 fractions to hypofractionated treatment to 51.6 Gy in 12 fractions for favorable risk prostate cancer showed similar low rates of late grade 3 or greater GI or GU toxicity with SBRT $(0.8 \%)$ or hypofractionated (1.7\%) treatment [20].

Similar to the limited toxicity in this study, follow-up extending out to 36 months post-treatment showed limited to no change in QOL as measured by either AUASS, IIEF scores, or EPIC-26 scores in either the urinary, bowel, sexual, or hormonal domains. There was a significant decline within the bowel domain of the EPIC-26 scores perhaps in concert with the slight increase in late, as compared to early, grade 2 or grade gastrointestinal. Similar increases in late bowel toxicity have been seen in phase III dose escalation studies of conventionally fractionated radiation and hypofractionated versus conventionally fractionated treatment. These results should be interpreted with caution however given the relatively low rate of events in this study and decline in responses to the QOL questionnaires such that less than a third of patients responded to the EPIC-26 questionnaire at 36 months.

It is tempting to comment on the disease status of these patients, however with a short median follow-up of 27.6 months, the ability to make inference on the efficacy of SBRT is limited. Longer follow-up is required for a more robust outcome analysis. If disease control is found to be similar with the observed low rates of toxicity, the option of decreasing treatment time for men with low to intermediate risk prostate cancer will have significant implications for patient convenience and cost.

This study only included men with low to intermediate risk disease and the PSA in those with a Gleason score of 7 was limited to 10 or less. Therefore the results of this study should not be extended to patients with higher risk disease who might require coverage of the seminal vesicles in the treatment volume or those patients treated with concurrent androgen deprivation therapy, both of which were not allowed on this study. In addition it is likely that for at least some men on this study there is little to no advantage to having undergone radiation as compared to active surveillance. The present results should therefore have limited impact on the decision to undergo treatment or not.

\section{Conclusions}

In summary for men with low to intermediate risk prostate cancer toxicity following SBRT is low and similar to conventionally fractionated and hypofractionated treatment with limited to no change in QOL. Further follow-up is warranted to address disease control after this treatment.

\section{Abbreviations}

AUASS: American Urological Association Symptom Scores; CTV: Clinical target volume: EPIC 26: Expanded Prostate Cancer Index Composite Short Form; IIEF: International Index of Erectile Function; IMRT: Intensity modulated radiotherapy; PTV: Planning target volume; QOL: Quality of life;

SBRT: Stereotactic body radiotherapy

\section{Acknowledgements}

The authors would like to acknowledge Joan Cahill for study coordination and data collection.

\section{Funding source}

The authors did not receive any funding/outside support for this study.

Availability of data and materials

The datasets generated during and/or analysed during the current study are not publicly available due to patient confidentiality but are available from the corresponding author on reasonable request.

\section{Author' contributions}

$\mathrm{MB}$ analyzed the data and wrote the manuscript. MP, RK, and ZV recruited patients to the study. JW helped design the study and wrote the manuscript. WRL designed the study, recruited patients, and wrote the manuscript. All authors reviewed the manuscript prior to its publication. All authors read and approved the final manuscript.

\section{Competing interests}

The authors declare that they have no competing interests.

\section{Consent for publication}

Written informed consent for publication of clinical images was obtained from the patient. A copy of the consent form is available for review by the Editor of this journal.

Ethics approval and consent to participate

Institutional review board approval was obtained at each center and all participants provided written informed consent before registration.

\section{Author details}

'Department of Radiation Oncology, Duke University Medical Center, DUMC Box 3085, Durham, NC 27710, USA. ${ }^{2}$ Coastal Carolina Radiation Oncology, Wilmington, NC, USA. ${ }^{3}$ Department of Radiation Oncology, Walter Reed National Military Medical Center, Bethesda, MD, USA. ${ }^{4}$ Department of Radiation Oncology, University of Maryland School of Medicine, Baltimore, MD, USA.

Received: 30 August 2016 Accepted: 28 December 2016

Published online: 13 January 2017

\section{References}

1. Dearnaley DP, Jovic G, Syndikus I, Khoo V, Cowan RA, Graham JD, Aird EG, Bottomley D, Huddart RA, Jose CC, et al. Escalated-dose versus control-dose 
conformal radiotherapy for prostate cancer: long-term results from the MRC RT01 randomised controlled trial. Lancet Oncol. 2014;15:464-73.

2. Kuban DA, Tucker SL, Dong L, Starkschall G, Huang EH, Cheung MR, Lee AK, Pollack A. Long-term results of the M. D. Anderson randomized dose-escalation trial for prostate cancer. Int J Radiat Oncol Biol Phys. 2008;70:67-74.

3. Peeters ST, Heemsbergen WD, Koper PC, van Putten WL, Slot A, Dielwart MF, Bonfrer JM, Incrocci L, Lebesque JV. Dose-response in radiotherapy for localized prostate cancer: results of the Dutch multicenter randomized phase III trial comparing 68 Gy of radiotherapy with 78 Gy. J Clin Oncol. 2006;24:1990-6.

4. Zietman AL, Bae K, Slater JD, Shipley WU, Efstathiou JA, Coen JJ, Bush DA, Lunt M, Spiegel DY, Skowronski R, et al. Randomized trial comparing conventional-dose with high-dose conformal radiation therapy in earlystage adenocarcinoma of the prostate: long-term results from proton radiation oncology group/american college of radiology 95-09. J Clin Oncol. 2010;28:1106-11.

5. Miralbell R, Roberts SA, Zubizarreta E, Hendry JH. Dose-fractionation sensitivity of prostate cancer deduced from radiotherapy outcomes of 5,969 patients in seven international institutional datasets: alpha/beta $=1.4$ (0.9-2 2) Gy. Int J Radiat Oncol Biol Phys. 2012;82:e17-24.

6. Shaffer R, Pickles T, Lee R, Moiseenko V. Deriving prostate alpha-beta ratio using carefully matched groups, long follow-up and the phoenix definition of biochemical failure. Int J Radiat Oncol Biol Phys. 2011;79:1029-36.

7. Alongi F, Cozzi L, Arcangeli S, Iftode C, Comito T, Villa E, Lobefalo F, Navarria P, Reggiori G, Mancosu P, et al. Linac based SBRT for prostate cancer in 5 fractions with VMAT and flattening filter free beams: preliminary report of a phase II study. Radiat Oncol. 2013;8:171.

8. Hannan R, Tumati V, Xie XJ, Cho LC, Kavanagh BD, Brindle J, Raben D, Nanda A, Cooley S, Kim DW, et al. Stereotactic body radiation therapy for low and intermediate risk prostate cancer-Results from a multi-institutional clinical trial. Eur J Cancer. 2016;59:142-51.

9. King CR, Brooks JD, Gill H, Pawlicki T, Cotrutz C, Presti Jr JC. Stereotactic body radiotherapy for localized prostate cancer: interim results of a prospective phase II clinical trial. Int J Radiat Oncol Biol Phys. 2009;73:1043-8.

10. Madsen BL, Hsi RA, Pham HT, Fowler JF, Esagui L, Corman J. Stereotactic hypofractionated accurate radiotherapy of the prostate (SHARP), $33.5 \mathrm{~Gy}$ in five fractions for localized disease: first clinical trial results. Int I Radiat Oncol Biol Phys. 2007;67:1099-105.

11. McBride SM, Wong DS, Dombrowski JJ, Harkins B, Tapella P, Hanscom HN, Collins SP, Kaplan ID. Hypofractionated stereotactic body radiotherapy in low-risk prostate adenocarcinoma: preliminary results of a multi-institutional phase 1 feasibility trial. Cancer. 2012;118:3681-90.

12. Tang Cl, Loblaw DA, Cheung P, Holden L, Morton G, Basran PS, Tirona R, Cardoso M, Pang G, Gardner S, Cesta A. Phase I/II study of a five-fraction hypofractionated accelerated radiotherapy treatment for low-risk localised prostate cancer: early results of pHART3. Clin Oncol (R Coll Radiol). 2008;20: 729-37.

13. Koontz BF, Tsivian M, Mouraviev V, Sun L, Vujaskovic Z, Moul J, Lee WR. Impact of primary Gleason grade on risk stratification for Gleason score 7 prostate cancers. Int J Radiat Oncol Biol Phys. 2012;82:200-3.

14. Barry MJ, Fowler Jr FJ, O'Leary MP, Bruskewitz RC, Holtgrewe HL, Mebust WK, Cockett AT. The American Urological Association symptom index for benign prostatic hyperplasia. The Measurement Committee of the American Urological Association. J Urol. 1992;148:1549-57. discussion 1564.

15. Rosen RC, Cappelleri JC, Smith MD, Lipsky J, Pena BM. Development and evaluation of an abridged, 5-item version of the International Index of Erectile Function (IIEF-5) as a diagnostic tool for erectile dysfunction. Int J Impot Res. 1999;11:319-26.

16. Wei JT, Dunn RL, Litwin MS, Sandler HM, Sanda MG. Development and validation of the expanded prostate cancer index composite (EPIC) for comprehensive assessment of health-related quality of life in men with prostate cancer. Urology. 2000;56:899-905.

17. Aluwini S, Pos F, Schimmel E, van Lin E, Krol S, van der Toorn PP, de Jager $H$, Dirkx M, Alemayehu WG, Heijmen B, Incrocci L. Hypofractionated versus conventionally fractionated radiotherapy for patients with prostate cancer (HYPRO): acute toxicity results from a randomised non-inferiority phase 3 trial. Lancet Oncol. 2015;16:274-83.

18. Lee WR, Dignam JJ, Amin MB, Bruner DW, Low D, Swanson GP, Shah AB, D'Souza DP, Michalski JM, Dayes IS, Seaward SA, Hall WA, Nguyen PL,
Pisansky TM, Faria SL, Chen Y, Koontz BF, Paulus R, Sandler HM. Randomized phase III noninferiority study comparing two radiotherapy fractionation schedules in patients with low-risk prostate cancer. J Clin Oncol. 2016; 34(20):2325-32. doi:10.1200/JCO.2016.67.0448. PubMed PMID: 27044935; PubMed Central PMCID:PMC4981980.

19. Wilkins A, Mossop H, Syndikus I, Khoo V, Bloomfield D, Parker C, Logue J, Scrase C, Patterson H, Birtle A, et al. Hypofractionated radiotherapy versus conventionally fractionated radiotherapy for patients with intermediate-risk localised prostate cancer: 2-year patient-reported outcomes of the randomised, non-inferiority, phase 3 CHHiP trial. Lancet Oncol. 2015;16: $1605-16$.

20. Lukka H, Stephanie P, Bruner D, Bahary JP, Lawton CAF, Efstathiou JA, Kudchadker R, Ponsky L, Seaward SA, Dayes IS, et al. Patient-reported outcomes in NRG Oncology/RTOG 0938, a randomized phase 2 study evaluating 2 Ultrahypofractionated Regimens (UHRs) for prostate cancer. Int J Radiat Oncol Biol Phys. 2016;94:2.

\section{Submit your next manuscript to BioMed Central and we will help you at every step:}

- We accept pre-submission inquiries

- Our selector tool helps you to find the most relevant journal

- We provide round the clock customer support

- Convenient online submission

- Thorough peer review

- Inclusion in PubMed and all major indexing services

- Maximum visibility for your research

Submit your manuscript at www.biomedcentral.com/submit
Biomed Central 